\title{
Review \\ Cognitive Rehabilitation in Multiple Sclerosis in the Period from 2013 and 2021: A Narrative Review
}

\author{
Bruno Brochet (D)
}

Citation: Brochet, B. Cognitive Rehabilitation in Multiple Sclerosis in the Period from 2013 and 2021: A

Narrative Review. Brain Sci. 2022, 12, 55. https://doi.org/10.3390/ brainsci12010055

Academic Editor: Omar Cauli

Received: 8 December 2021

Accepted: 27 December 2021

Published: 30 December 2021

Publisher's Note: MDPI stays neutral with regard to jurisdictional claims in published maps and institutional affiliations.

Copyright: (c) 2021 by the author Licensee MDPI, Basel, Switzerland. This article is an open access article distributed under the terms and conditions of the Creative Commons Attribution (CC BY) license (https:/ / creativecommons.org/licenses/by/ $4.0 /)$.
Neurocentre Magendie, INSERM U 1215, Université de Bordeaux, 33077 Bordeaux, France; bruno.brochet@u-bordeaux.fr

\begin{abstract}
Background: In recent years, several blinded randomized controlled trials (RCT) have been conducted on cognitive rehabilitation (CR) in adults with multiple sclerosis (MS). Objective: To review all blinded RCTs on CR in MS published since 2013. Methods: The National Library of Medicine database (Medline) and PSYCINFO were searched using the keywords MS and CR or cognitive training or NP rehabilitation or memory rehabilitation or attention rehabilitation. Results: After the exclusion of some papers not specifically focused on CR, a final list of 26 studies was established. The papers belong to three main categories: individual specific rehabilitation (8studies), group rehabilitation (4 studies), and computerized training (CT) (14 studies), while one study combined group rehabilitation and CT. Among the individual rehabilitation studies, 5 were devoted to memory, and most of the 19 other selected studies were about several cognitive domains. Most of the studies mainly concerned RRMS patients, except for 2 studies that were carried out exclusively in progressive forms. Despite the methodological limitations of some studies and the great heterogeneity of the protocols, the results are generally in favor of the efficacy of CR in neuropsychological tests. Conclusion: Recent blinded RCTs about CR in MS show promising results.
\end{abstract}

Keywords: multiple sclerosis; cognitive rehabilitation; cognition; memory; review

\section{Introduction}

Treating cognitive impairment (CI) in multiple sclerosis (MS), the leading cause of disability due to nontraumatic neurological disease in young adults, is an important challenge [1]. The contribution of CI to disability in MS has been increasingly recognized, and $\mathrm{CI}$ has been shown to decrease health-related quality of life (HR-QoL), even in the early stages of the disease [2]. CI negatively affects daily activities such as driving, vocational status, absenteeism, and instrumental activities in persons living with MS [3]. No medication has proven to have a consistent symptomatic effect on CI in MS, and disease-modifying therapies only have a limited impact on CI progression [4].

$\mathrm{CI}$ in MS is dominated by a slowdown in information processing speed (IPS), as well as by disturbances of more specific cognitive functions such as attention, episodic memory (EM), working memory (WM), and executive function (EF) [5]. If a relatively circumscribed alteration in IPS linked to a specific process deficit can occur, changes in IPS can alter other cognitive processes and usually reflect cognitive functioning and efficiency. The alteration of IPS has consequences for WM, attention, EF, and EM. IPS impairment predicts later disability vocational status and changes in quality of life [3].

Cognitive rehabilitation (CR) is the most promising approach for treating MS-related CI, despite important methodological shortcomings. CR programs could include techniques designed to improve specific domains of cognitive function such as memory, attention, or executive functions, but they can also include psychotherapy targeting emotional symptoms, behavioral interventions, and interventions targeting psychomotor issues such as motor-cognitive interference. In this review, we will focus only in interventions designed to improve specific cognitive domains in MS. 
A wide variety of neuropsychological tests have been used in the assessment of cognitive impairment in MS. A panel of experts recently proposed recommendations [3] for screening tests—-the Symbol Digit Modalities Test (SDMT) [6], the Processing Speed Test (PST) [7], and the Computerized Speed Cognitive Test (CSCT) [8], a self-report scale (Multiple Sclerosis Neuropsychological Screening Questionnaire (MSNQ) [9]—and recommended brief cognitive batteries: the Brief International Cognitive Assessment for Multiple Sclerosis (BICAMS) [10], the Brief Repeatable Neuropsychological Battery (BRNB) [11], and the Minimal Assessment of Cognitive Function in MS (MACFIMS) [12]. While most of the CR studies used the SDMT, the other assessment tests varied widely from study to study.

In 2013, Amato et al. reviewed the available studies at that time on CR and listed 23 studies [1]. However, methodological limitations in many studies have led to disappointing results, and well-designed studies are still scarce, as shown by another contemporary review [13]. Many studies lack a randomized controlled design that includes passive or active control conditions, primary neuropsychological (NP) endpoints identified a priori, evidence of the sustainability of $\mathrm{CR}$, and the inclusion of near and far transfer outcomes. Tertiary outcomes of QoL, metacognition, or other patient-reported outcomes (PROs) have rarely been used. Since this time, many studies have been performed, and many were randomized controlled trials (RCT). We review here the RCTs with blinded assessments that have been published between 2013 and 2021.

\section{Methods}

The National Library of Medicine database (Medline) was searched at https: / pubmed. ncbi.nlm.nih.gov/ using the following keywords on 18 November 2021.

MS and CR or cognitive training or NP rehabilitation or memory rehabilitation or attention rehabilitation.

Inclusion criteria were as follows: RCTs, published in English as full papers, blinded assessment, cognitive intervention (excluding exercise training and cognitive and motor joint intervention such as dual tasks and intervention combined with brain stimulation, music therapy, etc.), specified endpoint focused on cognitive outcomes (NP testing required) (excluding studies focused on fatigue, motor outcome, depression, and anxiety as primary outcomes, feasibility studies, and reports devoted primarily to imaging outcomes). Quantitative results should be presented in the paper, excluding papers about research protocols and qualitative studies. Studies in pediatric-onset MS were not included.

\subsection{Results}

A first search with the terms multiple sclerosis and CR, RCT, 2013-2021, full text resulted in a list of 141 references. A similar search was performed on the PSYCINFO database and resulted in a list of 31 references. After application of the eligibility criteria, 23 papers were selected. After running searches using the other pre-selected keywords (memory rehabilitation and MS), three additional papers were selected. Table 1 presents the list of the 26 studies included in the analysis [14-39]. Papers were classified into three main categories: individual specific rehabilitation (eight studies), group rehabilitation (four studies), and other computerized training (CT) (14 studies), while one study combined group rehabilitation and CT [30]. Most of the studies mainly concern Relapsing-Remitting MS (RRMS) patients, with the exception of two studies carried out exclusively in progressive forms $[33,36]$. Five studies investigated memory rehabilitation exclusively, while the other studies addressed multiple domains.

\subsection{Methodological Analysis}

All studies were blinded RCTs. Thirteen studies were double blinded and thirteen were single blinded (blinded evaluators) (Table 1). Some bias can be identified. Three studies included patients regardless of the presence of $\mathrm{CI}[19,24,30]$. We did not consider this as an exclusion criterion in our review, but this may have affected the results of these studies. In nine studies, the control group did not have any intervention (waiting list or usual care). 
However, in the other 17 studies, a placebo or sham intervention was administered in the control group. Several studies were considered pilot studies and thus, their power was limited. The sample size was less than 35 subjects in nine studies and more than 100 subjects in only four studies (Table 1).

Table 1. Selected studies.

\begin{tabular}{|c|c|c|c|c|c|c|c|}
\hline Study & Blinding & MS Types (\%) & CI & $\mathbf{n}$ & $\begin{array}{l}\text { Cognitive } \\
\text { Domains }\end{array}$ & Type & Setting \\
\hline Chiaravalloti et al. (2013) [14] & double & $\begin{array}{c}\text { RR (64); SP (20), } \\
\text { PP (6) }\end{array}$ & + & 86 & Memory & Individual & Institution \\
\hline Cerasa et al. (2013) [15] & double & RR (100) & + & 26 & Attention & $\mathrm{CT}$ & Institution \\
\hline Rosti-Otajärvi et al. (2013) [16] & single & RR (100) & + & 99 & Attention & Individual & Institution \\
\hline Amato et al. (2014) [17] & double & RR (100) & + & 88 & Attention & $\mathrm{CT}$ & Home \\
\hline De Giglio et al. (2015) [18] & single & RR (100) & + & 35 & $\begin{array}{c}\text { Memory, } \\
\text { attention, } \\
\text { visuospatial } \\
\text { processing and } \\
\text { calculations }\end{array}$ & $\mathrm{CT}$ & Home \\
\hline Hancock et al. (2015) [19] & double & $\begin{array}{c}\text { RR (70), SP (17), } \\
\text { PP (13) }\end{array}$ & - & 40 & IPS and WM & $\mathrm{CT}$ & Home \\
\hline Gich et al. (2015) [20] & single & RR (88), SP (22) & + & 43 & Multiple & Individual & Institution \\
\hline Pedullà et al. (2016) [21] & single & RR (61), SP (39) & + & 28 & $\mathrm{WM}$ & $\mathrm{CT}$ & Home \\
\hline Campbell et al. (2016) [22] & double & RR (70), SP (30) & + & 35 & $\begin{array}{l}\text { WM, memory, } \\
\text { attention }\end{array}$ & $\mathrm{CT}$ & Home \\
\hline Charvet et al. (2017) [23] & double & RR (100) & + & 135 & $\begin{array}{l}\text { IPS, attention, } \\
\text { WM, EF }\end{array}$ & $\mathrm{CT}$ & TR \\
\hline Grasso et al. (2017) [24] & single & $\begin{array}{c}\text { RR (47), SP (44), } \\
\text { PP (9) }\end{array}$ & - & 34 & attention, IPS, EF & $\mathrm{CT}$ & Institution \\
\hline Messinis et al. (2017) [25] & single & RR (100) & + & 58 & $\begin{array}{l}\text { Memory, IPS, } \\
\text { attention, EF }\end{array}$ & $\mathrm{CT}$ & Institution \\
\hline Chiaravalloti et al. (2018) [26] & double & RR (100) & + & 21 & IPS & $\mathrm{CT}$ & Institution \\
\hline Goverover et al. (2018) [27] & double & $\begin{array}{c}\text { RR (69), SP (11), } \\
\text { PP (20) }\end{array}$ & + & 35 & Memory & Individual & Institution \\
\hline Mani et al. (2018) [28] & single & Not specified & + & 34 & $\begin{array}{l}\text { Memory, } \\
\text { attention, EF }\end{array}$ & Group & Institution \\
\hline Moussavi et al. (2018) [29] & double & Not specified & + & 60 & WM & Group & Institution \\
\hline Stuifbergen et al. (2018) [30] & single & RR (68) & - & 183 & $\begin{array}{c}\text { Memory, } \\
\text { attention, } \\
\text { problem solving }\end{array}$ & Group $+\mathrm{CT}$ & Institution/Home \\
\hline Krch et al. (2019) [31] & double & RR (100) & + & 20 & Memory & Individual & Institution \\
\hline Brissart et al. (2020) [32] & double & RR (78) & + & 110 & $\begin{array}{l}\text { Memory, EF, } \\
\text { language }\end{array}$ & Group & Institution \\
\hline Chiaravalloti et al. (2020) [33] & double & SP (57), PP (39) & + & 30 & Memory & Individual & Institution \\
\hline Lamargue et al. (2020) [34] & single & $\begin{array}{c}\text { RR (83), SP (11) } \\
\text { PP (6) }\end{array}$ & + & 35 & $\begin{array}{l}\text { Attention, IPS, } \\
\text { EF, WM }\end{array}$ & Individual & Real life \\
\hline Lincoln et al. (2020) [35] & single & $\begin{array}{c}\text { RR (65), SP (25), } \\
\text { PP (10) }\end{array}$ & + & 449 & $\begin{array}{l}\text { Attention and } \\
\text { memory }\end{array}$ & Group & Institution \\
\hline Messinis et al. (2020) [36] & single & SP (100) & + & 36 & $\begin{array}{l}\text { Memory, IPS, } \\
\text { attention, EF }\end{array}$ & $\mathrm{CT}$ & Home \\
\hline Vilou et al. (2020) [37] & single & RR (100) & + & 47 & $\begin{array}{c}\text { Memory, } \\
\text { attention, IPS }\end{array}$ & $\mathrm{CT}$ & TR \\
\hline Chiaravalloti et al. (2021) [38] & double & $\begin{array}{c}\text { RR (65), SP (10), } \\
\text { PP (15) }\end{array}$ & + & 20 & Memory & Individual & Institution \\
\hline Blair et al. (2022) [39] & single & $\begin{array}{l}\text { RR (57), SP } \\
\text { (40);PP (3) }\end{array}$ & + & 30 & WM & $\mathrm{CT}$ & TR \\
\hline
\end{tabular}

CI: cognitive impairment required in inclusion criteria; CT: computerized training; EF: executive functions; IPS information processing speed; $\mathrm{n}$ = sample size; RR: relapsing-remitting; SP: secondary progressive; PP: primary progressive; TR: telerehabilitation WM: working memory. 
Table 2 summarizes the outcomes used in these studies.

Table 2. Outcomes.

\begin{tabular}{|c|c|}
\hline Batteries & $\begin{array}{l}\text { Addenbrooke's cognitive examination (ACE); Brief-Repeatable Battery (BRB); } \\
\text { Wechsler Adult Intelligence Scale (WAIS) }\end{array}$ \\
\hline Episodic memory tests & $\begin{array}{l}\text { Brief Visuospatial Memory Test-Revised (BVMT-R); California Verbal Learning } \\
\text { Test-2nd edition (CVLT-II); Contextual Memory Test (CMT); Greek Verbal Learning } \\
\text { Test (GVLT); Hopkins Verbal Learning Test-Revised (HVLT-R); Memory Assessment } \\
\text { Scales, Prose Memory (MAS); Memory for Intentions Test (MIST); Memory } \\
\text { Functioning Questionnaire (MFQ); Rivermead Behavioral Memory Test (RBMT); } \\
\text { Spatial Recall Test (SPART; 10-36); Wechsler Memory Scale-Revised (WMS-R) }\end{array}$ \\
\hline Information processing speed tests & Symbol Digit Modalities Test (SDMT) \\
\hline Attention/Working memory & $\begin{array}{l}\text { Letter-Number Sequencing (LNS); Test of Attentional Performance (TAP); Continuous } \\
\text { Performance Test (CPT); Paced Auditory Serial Addition Test (PASAT) }\end{array}$ \\
\hline Executive functions & $\begin{array}{l}\text { Test of Attentional Performance (TAP); Trail-Making Test (TMT); Word List Generation } \\
\text { (WLG); Behavior Rating Inventory of Executive Function-Adult (BRIEF-A); } \\
\text { Controlled Oral Word Association Test (COWAT); Wisconsin Card-Sorting Test } \\
\text { (WCST) }\end{array}$ \\
\hline Other & Boston Naming Test (BNT) \\
\hline Ecological assessment & $\begin{array}{l}\text { Actual Reality (AR); Urban DailyCog; Timed Instrumental Activities of Daily Living } \\
\text { Test (TIADL) }\end{array}$ \\
\hline Quality of Life scales & $\begin{array}{l}\text { Functional Assessment of Multiple Sclerosis (FAMS); Mental Health Inventory (MHI); } \\
\text { Short Form survey-36 (SF-36); Satisfaction with Life Scale (SWLS); Brief version of the } \\
\text { World Health Organization Quality of Life (WHOQoL-BREF); Multiple Sclerosis } \\
\text { Impact Scale, psychological (MSIS-psy); Multiple Sclerosis Quality of Life Inventory } \\
\text { (MSQLI); EuroQoL five-dimension questionnaire (EQ-5D); MS Quality of Life } \\
\text { questionnaire (MSQoL54) }\end{array}$ \\
\hline Other self-report scales & $\begin{array}{l}\text { Daily Cognitive Activities Questionnaire (DCAQ); Frontal Systems Behavior Scale } \\
\text { (FrSBe); Perceived Deficits Questionnaire (PDQ); Self-Regulation Skills Interview } \\
\text { (SRSI); Everyday Memory Questionnaire (EMQ); Everyday Problems Test-Revised } \\
\text { (EPT-R); Memory Functioning Questionnaire (MFQ); PROMIS (self-reported cognitive } \\
\text { function) }\end{array}$ \\
\hline
\end{tabular}


Table 3. Blinded RCT about individual-specific cognitive rehabilitation.

\begin{tabular}{|c|c|c|c|c|c|c|c|c|c|c|}
\hline 1st Author & Intervention & $\begin{array}{l}\text { Session Duration } \\
\text { (Min) }\end{array}$ & $\begin{array}{l}\text { CR Du- } \\
\text { ration } \\
\text { (Weeks) }\end{array}$ & $\begin{array}{c}\text { Total } \\
\text { Duration } \\
\text { (Min) }\end{array}$ & $\begin{array}{c}\text { Control } \\
\text { Intervention }\end{array}$ & $\begin{array}{l}\text { Main Positive Results } \\
\text { (Primary Outcomes) }\end{array}$ & LTFU (Mths) & $\begin{array}{c}\text { Positive } \\
\text { Results } \\
\text { (Secondary } \\
\text { Outcomes) }\end{array}$ & $\begin{array}{l}\text { Other } \\
\text { Positive } \\
\text { Results }\end{array}$ & $\begin{array}{l}\text { Main Negative } \\
\text { Results (Primary } \\
\text { Outcomes } \\
\text { Underlined) }\end{array}$ \\
\hline \multicolumn{11}{|c|}{ Episodic Memory } \\
\hline $\begin{array}{l}\text { Chiaravalloti } \\
\text { et al. [14] }\end{array}$ & m-SMT & $\begin{array}{c}\text { 45-60 min twice a } \\
\text { week }\end{array}$ & 5 & $450 / 600$ & Placebo & CVLT-II, RBMT & 6 & FAMS & & $\begin{array}{l}\text { No effect of booster } \\
\text { sessions }\end{array}$ \\
\hline $\begin{array}{l}\text { Goverover } \\
\text { et al. [27] }\end{array}$ & Self-GEN & $\begin{array}{c}60 \text { min twice a } \\
\text { week }\end{array}$ & 3 & 360 & Placebo & CMT, SRSI & ND & MIST, FAMS & & CVLT-II, MFQ, AR \\
\hline $\begin{array}{l}\text { Chiaravalloti } \\
\text { et al. [33] }\end{array}$ & m-SMT & $\begin{array}{c}45-60 \text { min twice a } \\
\text { week }\end{array}$ & 5 & $450 / 600$ & Placebo & CVLT-II & 3 & FrSBe & & $\begin{array}{l}\text { RBMT; BVMT-R; MAS, } \\
\text { FAMS }\end{array}$ \\
\hline $\begin{array}{l}\text { Chiaravalloti } \\
\text { et al. [38] }\end{array}$ & STEM & $\begin{array}{c}\text { 30-45 min twice a } \\
\text { week }\end{array}$ & 4 & $240 / 360$ & Placebo & - & & $\begin{array}{c}\text { SF-36, } \\
\text { FAMS, MHI }\end{array}$ & & $\underline{\text { CVLT }}$ \\
\hline \multicolumn{11}{|c|}{ Multidomain } \\
\hline $\begin{array}{l}\text { Rosti-Otajärvi } \\
\text { et al. [16,40] }\end{array}$ & NP intervention & $\begin{array}{c}60 \text { min once a } \\
\text { week }\end{array}$ & 13 & 780 & No & $\underline{\mathrm{PDQ}}$ & 12 & TMT-A & & $\begin{array}{l}\text { WHOQoL-BREF, } \\
\text { SDMT }\end{array}$ \\
\hline $\begin{array}{l}\text { Lamargue } \\
\text { et al. [34] }\end{array}$ & REACTIV & $\begin{array}{c}45 \text { min, } 3 \text { times a } \\
\text { week }\end{array}$ & & 2250 & $\begin{array}{l}\text { Non-specific } \\
\text { training }\end{array}$ & TAP (Alertness, Divided Attention) & 4 & $\begin{array}{c}\text { CVLT, Rey } \\
\text { figure copy; } \\
\text { Urban } \\
\text { Daily-Cog, } \\
\text { DCAQ }\end{array}$ & & $\underline{\text { SDMT *, n-Back, TMT }}$ \\
\hline
\end{tabular}

CR: cognitive rehabilitation; Min: minutes; LTFU: positive results at long term follow-up; ND: not done; Mths: months; *: test improved in both groups. Rehabilitation programs m-SMT: modified-story memory technique; Self-Gen: self-generation learning program; STEM: Strategy-based Training to Enhance Memory; MS-Line!: NP intervention using written, manipulative, and computer-based materials; REACTIV: NP intervention using written and computer-based materials. Outcomes: AR: Actual Reality; BNT: Boston Naming Test; BVMT-R: Brief Visuospatial Memory Test—Revised; CMT: Contextual Memory Test; CVLT-II: California Verbal Learning Test—2nd edition; DCAQ: Daily Cognitive Activities Questionnaire. FAMS: Functional Assessment of Multiple Sclerosis; FrSBe: Frontal Systems Behavior Scale; HVLT-R: Hopkins Verbal Learning Test—Revised; MAS: Memory Assessment Scales, Prose Memory; MIST: Memory for Intentions Test; MFQ: Memory Functioning Questionnaire; MHI: Mental Health Inventory; LNS: Letter-Number Sequencing. PDQ: Perceived Deficits Questionnaire; RBMT: Rivermead Behavioral Memory Test; SDMT: Symbol Digit Modalities Test; SF-36: Short Form survey-36; SPART: Spatial Recall Test (10-36); SRSI: Self-Regulation Skills Interview; SWLS: Satisfaction with Life Scale; TAP: Test of Attentional Performance; TMT: Trail-Making Test; Urban DailyCog: Ecological test in a virtual realty environment; WHOQoL-BREF = Brief version of the World Health Organization Quality of Life; WLG: Word List Generation. Underlined outcomes are primary outcomes. 
Table 4. Group Interventions blinded RCT.

\begin{tabular}{|c|c|c|c|c|c|c|c|c|c|c|}
\hline 1st Author & Intervention & $\begin{array}{l}\text { Session Duration } \\
\text { (Min) }\end{array}$ & $\begin{array}{c}\text { CR } \\
\text { Duration } \\
\text { (Weeks) }\end{array}$ & $\begin{array}{l}\text { Total } \\
\text { Duration } \\
\text { (Min) }\end{array}$ & $\begin{array}{c}\text { Control } \\
\text { Intervention }\end{array}$ & $\begin{array}{l}\text { Main Positive Results } \\
\text { (Primary Outcomes) }\end{array}$ & LTFU (Mths) & $\begin{array}{c}\text { Positive } \\
\text { Results } \\
\text { (Secondary } \\
\text { Outcomes) }\end{array}$ & $\begin{array}{l}\text { Other } \\
\text { Positive } \\
\text { Results }\end{array}$ & $\begin{array}{l}\text { Main Negative } \\
\text { Results (Primary } \\
\text { Outcomes } \\
\text { Underlined) }\end{array}$ \\
\hline \multicolumn{11}{|c|}{ Multidomain } \\
\hline $\begin{array}{l}\text { Mani et al. } \\
\text { [28] }\end{array}$ & $\begin{array}{l}\text { Group + } \\
\text { homework }\end{array}$ & $\begin{array}{c}120 \text { min twice a } \\
\text { week }\end{array}$ & 4 & 960 & $\begin{array}{l}\text { No training } \\
\text { (sham } \\
\text { intervention) }\end{array}$ & $\begin{array}{c}\text { No specified primary } \\
\text { outcome }\end{array}$ & 4 & & $\begin{array}{c}\text { ACE } \\
\text { (memory), } \\
\text { BRIEF-A, } \\
\text { MFQ WCST, } \\
\text { WMS-R }\end{array}$ & CPT (attention) \\
\hline $\begin{array}{c}\text { Stuifbergen et al. } \\
{[30]}\end{array}$ & $\begin{array}{l}\text { Group + } \\
\text { CT at home } \\
\text { (Lumosity) }\end{array}$ & $\begin{array}{c}120 \text { min once a } \\
\text { week }+ \\
45 \text { min, } 3 \text { times a } \\
\text { week }\end{array}$ & 8 & $\begin{array}{l}\text { Variable Up to } \\
2040\end{array}$ & $\begin{array}{l}\text { No intervention } \\
\text { (games) }\end{array}$ & - & 6 & $\begin{array}{l}\text { CVLT, } \\
\text { PASAT }\end{array}$ & & $\begin{array}{c}\text { EPT-R, BVMT-R, } \\
\text { COWAT, SDMT, } \\
\text { PROMIS }\end{array}$ \\
\hline $\begin{array}{c}\text { Brissart et al. } \\
\text { [32] }\end{array}$ & Group & $\begin{array}{l}120 \text { min twice a } \\
\text { month }\end{array}$ & 24 & 1560 & $\begin{array}{l}\text { No training } \\
\text { (placebo } \\
\text { intervention) }\end{array}$ & SRT (learning index) & ND & $\begin{array}{l}\text { Digit span } \\
\text { backward, } \\
\text { TAP (WM) }\end{array}$ & & $\begin{array}{c}\text { Flexibility (TAP), } \\
\text { verbal fluency, IPS } \\
\text { (WAIS digit-symbol), } \\
\text { SPART; MSQLI }\end{array}$ \\
\hline $\begin{array}{c}\text { Lincoln et al. } \\
{[35]}\end{array}$ & $\begin{array}{l}\text { Group (4-6 } \\
\text { persons) }\end{array}$ & 90 min each week & 10 & 900 & No intervention & - & 12 & $\begin{array}{l}\text { EMQ MSIS } \\
\text { (6Mths), }\end{array}$ & & $\frac{\text { MSIS-psy (12Mths), }}{\text { BRB tests }}$ \\
\hline
\end{tabular}

CR: cognitive rehabilitation; Min: minutes; LTFU: positive results at long term follow-up; ND: not done; NP: neuropsychological; W: week; Mths: months; IPS: information processing speed; WM: working memory. Outcomes: ACE. Adde BVMT-R: Bief Visusatil Memory Test-Revised, COWAT: Contolled Oral Word Association Test: CPT: Continuous Performance Test: CVLT-II: California Verbal Learning BVMT-R: Bref Test-2nd edition, EMQ: Everyday Memory Questionnaire EPT-R: Everyday Problems Test-Revised; MFQ: Memory Functioning Questionnaire; MSIS-psy: Multiple Sclerosis Impact Scale, psychological; MSQLI: Mest Modalities Test; SPART: Spatial Recall Test (10-36); SRT: Selective Reminding Test; TAP: Test of Attentional Performance; WAIS: Wechsler Adult Intelligence Scale; WCST: Wisconsin Card-Sorting Test; WMS-R: Wechsler Memory Scale-Revised. Underlined outcomes are primary outcomes. 
Table 5. Blinded RCT of CT in MS.

\begin{tabular}{|c|c|c|c|c|c|c|c|c|c|c|c|}
\hline 1st Author & Year & $\begin{array}{l}\text { Intervention } \\
\text { (Software) }\end{array}$ & $\begin{array}{c}\text { Session } \\
\text { Duration (Min) }\end{array}$ & $\begin{array}{l}\text { CR Duration } \\
\text { (Weeks) }\end{array}$ & $\begin{array}{l}\text { Total Duration } \\
\text { (Min) }\end{array}$ & $\begin{array}{c}\text { Control } \\
\text { Intervention }\end{array}$ & $\begin{array}{l}\text { Main Positive } \\
\text { Results (Primary } \\
\text { Outcomes) }\end{array}$ & LTFU (Mths) & $\begin{array}{c}\text { Positive } \\
\text { Results } \\
\text { (Secondary } \\
\text { Outcomes) }\end{array}$ & $\begin{array}{l}\text { Other Positive } \\
\text { Results }\end{array}$ & $\begin{array}{l}\text { Main Negative Results } \\
\text { (Primary Outcomes } \\
\text { Underlined) }\end{array}$ \\
\hline $\begin{array}{l}\text { Cerasa } \\
\text { et al. [15] }\end{array}$ & 2013 & RehaCom & $\begin{array}{c}60 \begin{array}{c}\text { min twice a } \\
\text { week }\end{array} \\
\end{array}$ & 6 & 720 & Placebo training & $\begin{array}{c}\text { no specified } \\
\text { primary outcome }\end{array}$ & ND & & ST & BRB tests, TMT \\
\hline $\begin{array}{l}\text { Amato } \\
\text { et al. [17] }\end{array}$ & 2014 & APT & $\begin{array}{c}60 \text { min twice a } \\
\text { week }\end{array}$ & 12 & 1440 & Placebo training & $\begin{array}{c}\text { no specified } \\
\text { primary outcome }\end{array}$ & 6 & & PASAT & SDMT * \\
\hline $\begin{array}{l}\text { De Giglio } \\
\text { et al. [18] }\end{array}$ & 2015 & DKBT & $\begin{array}{c}30 \text { min } 3 \text { times a } \\
\text { week }\end{array}$ & 8 & 720 & No intervention & $\begin{array}{c}\text { no specified } \\
\text { primary outcome }\end{array}$ & ND & & $\begin{array}{l}\text { ST, SDMT, some } \\
\text { MSQoL54 } \\
\text { subscales }\end{array}$ & PASAT \\
\hline $\begin{array}{l}\text { Pedullà } \\
\text { et al. [21] }\end{array}$ & 2016 & Cogni-Track & $\begin{array}{c}30 \text { min } 5 \text { times a } \\
\text { week }\end{array}$ & 8 & 1200 & $\begin{array}{l}\text { Non-adaptative } \\
\text { training }\end{array}$ & $\begin{array}{c}\text { no specified } \\
\text { primary outcome }\end{array}$ & 6 & & $\begin{array}{l}\text { SRT, SDMT, } \\
\text { PASAT, WLG }\end{array}$ & SPART, WCST \\
\hline $\begin{array}{l}\text { Campbell } \\
\text { et al. [22] }\end{array}$ & 2016 & RehaCom & $\begin{array}{c}45 \text { min } 3 \text { times a } \\
\text { week }\end{array}$ & 6 & 810 & Placebo training & $\underline{\text { SDMT }}$ & 3 & & & 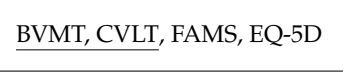 \\
\hline $\begin{array}{l}\text { Charvet } \\
\text { et al. [23] }\end{array}$ & 2017 & Brain HQ (PS) & $\begin{array}{c}60 \text { min } 5 \text { times a } \\
\text { week }\end{array}$ & 12 & 1800 & Placebo training & Composite NP score & ND & & & \\
\hline $\begin{array}{l}\text { Grasso } \\
\text { et al. [24] }\end{array}$ & 2017 & APT & $\begin{array}{c}60 \text { min } 3 \text { times a } \\
\text { week }\end{array}$ & 12 & 2160 & No intervention & $\begin{array}{c}\text { no specified } \\
\text { primary outcome }\end{array}$ & 6 & $* *$ & & \\
\hline $\begin{array}{l}\text { Chiaravalloti } \\
\text { et al. [26] }\end{array}$ & 2018 & SPT & $\begin{array}{c}30 \text { min twice a } \\
\text { week }\end{array}$ & 5 & 300 & No intervention & & ND & TIADL & & WAIS digit symbol $^{*}$ \\
\hline $\begin{array}{l}\text { Messinis } \\
\text { et al. [36] }\end{array}$ & 2020 & RehaCom & $\begin{array}{c}45 \text { min } 3 \text { times a } \\
\text { week }\end{array}$ & 8 & 1080 & Placebo training & $\underline{\text { SDMT, GVLT, BVMT }}$ & ND & EQ-5D & & \\
\hline $\begin{array}{c}\text { Vilou } \\
\text { et al. [37] }\end{array}$ & 2020 & Brain HQ (PS) & $\begin{array}{c}40 \text { min twice a } \\
\text { week }\end{array}$ & 6 & 480 & No intervention & $\begin{array}{c}\text { no specified } \\
\text { primary outcome }\end{array}$ & ND & & $\begin{array}{l}\text { GVLT, BVMT, } \\
\text { TMT-A, ST }\end{array}$ & SDMT \\
\hline $\begin{array}{c}\text { Blair } \\
\text { et al. [39] }\end{array}$ & 2021 & Cogmed & $\begin{array}{r}30-45 \min 5 \\
\text { times a week }\end{array}$ & 5 & $750-1125$ & No intervention & & 6 & & & PASAT *, SDMT *, ST * \\
\hline \multicolumn{12}{|c|}{$\begin{array}{l}\text { CR: cognitive rehabilitation; Min: minutes; LTFU: positive results at long-term follow-up; ND: not done; Mths: months; EM: episodic memory; IPS: information processing speed } \\
\text { *: test improved in both groups; ** no difference between groups. Rehabilitation programs: ATP: Attention Processing Training program; DKBT: Dr. Kawashima's Brain Training } \\
\text { (Nintendo); PS: Posit Science; SPT: Speed of Processing Training. Outcomes: BRB: Brief-Repeatable Battery; BVMT-R: Brief Visuospatial Memory Test-Revised; CVLT-II: California } \\
\text { Verbal Learning Test-2nd edition; EQ-5D: EuroQoL five-dimension questionnaire; FAMS: Functional Assessment of Multiple Sclerosis; GVLT: Greek Verbal Learning Test; LNS } \\
\text { Letter-Number Sequencing; MSQoL54: MS Quality of Life questionnaire; PASAT: Paced Auditory Serial Addition Test; SDMT: Symbol Digit Modalities Test; SPART: Spatial Recall Test } \\
\text { (10-36); SRT: Selective Reminding Test; ST: Stroop test; TIADL: Timed Instrumental Activities of Daily Living Test; TMT: Trail-Making Test. WAIS: Wechsler Adult Intelligence Scale } \\
\text { WCST: Wisconsin Card-Sorting Test; WLG: Word List Generation. Underlined outcomes are primary outcomes. }\end{array}$} \\
\hline
\end{tabular}


All studies included NP scores as outcomes (which were primary outcomes in 14 studies). Other primary outcomes were used in three studies: perceived deficits in one study $[16,40]$, instrumental activities of daily living (IADL) in one study [30], and HR-Qol in one study [35]. Nine reports did not identify a primary endpoint (Tables 3-5).

Most studies incorporated HRQoL measures in their outcomes, but ten did not $[15,17,21,23,25,26,28-30,37]$. Seventeen studies used some self-assessment of cognitive functioning as outcomes, but nine did not [15,19-21,25,29,32,36,37].

\section{Reported Results}

Tables 3-5 summarized the results of the selected studies. We considered as positive (for primary and secondary outcomes) only a superiority of the group receiving investigational treatment as compared to the control group (with no intervention or placebo/sham intervention) confirmed by ANOVA, ANCOVA, the Mann-Whitney U test, or some equivalent. In some instances, ${ }^{*}$ in the negative results column indicates results that show no difference between the treatment group and the control group, while there was a significant improvement in the treatment group and sometimes in the control group compared to the baseline evaluation.

\subsection{Individual-Specific Rehabilitation}

This category includes five studies on memory rehabilitation $[7,20,24,26,31]$ and three studies on several domains $[16,20,34]$ (Table 3$)$. The rehabilitation was carried out by a research assistant or a trainer in four studies $[14,31,32,38]$, a neuropsychologist in one study [9], speech therapists in one study [34], an occupational therapist in one study [27], and the therapist was not specified in one study [20]. The material used was generally written material, but it could include computer-assisted exercises (three studies) [20,31,34].

\subsubsection{Memory}

All studies devoted to the rehabilitation of episodic memory came from the same research group of the Kessler Foundation. After an initial pilot study dedicated to the modified-story memory technique (mSMT)-based rehabilitation method [41], which is basically an imagery- and context-based memory retraining program, Chiaravalloti et al. [14] conducted a pivotal study on a larger sample (86 patients) with a positive result on the main criterion, the learning slope of an NP test of EM (California Verbal Learning Testsecond edition, CVLT-II), and a positive effect on Functional Assessment of MS (FAMS), an assessment of HR-QoL, as a secondary endpoint. These positive results were maintained at the remote evaluation performed six months after treatment. The second primary endpoint assessing everyday objective memory (RBMT) was also significantly more improved in the treated group than in the control group but with a small effect size. This study did not show any efficacy of booster sessions.

Two other studies were devoted to the m-SMT technique with smaller sample sizes. One study concerned the Spanish adaptation of m-SMT performed in Mexico on 20 patients [31]. The primary endpoint (learning at the Hopkins Verbal Learning Test-Revised, an NP test of EM) was reached, and a secondary outcome about life satisfaction (Satisfaction with Life Scale) was also significantly improved. However, there was no significant improvement in the subjective perception of memory for the Memory Functioning Questionnaire (MFQ). A recent study evaluated the effectiveness of m-SMT in a sample of 30 patients with progressive forms of the disease [26]. The learning curve of the CVLT-II was significantly better in the treatment group than in the control group (primary endpoint), and a significant improvement was also noted on the Frontal Systems Behavior Scale, which is a measure of daily life functioning. However, the second primary endpoint (RBMT) was not reached, and other EM measures — the Brief Visuospatial Memory Test-Revised (BVMT-R) and the Memory Assessments Scales (prose memory) were not significantly improved, and neither was the FAMS (HR-QoL). 
Assuming that cognitive training focused on improving new learning via memoryenhancing techniques does not transfer to tasks other than those involved in the intervention and considering that items self-generated by an individual are better remembered than provided information, another method of memory rehabilitation was studied by this group, the self-generation learning program (Self-GEN) [27]. In this program, the therapist teaches patients to discover the benefit of using the self-generation strategy during training, and participants must discuss how the strategies could be used in other tasks. The study involved $35 \mathrm{MS}$ patients and reached primary endpoints (the Contextual Memory Test, an objective learning and memory test, and the Self-Regulation Skills Interview that assesses self-awareness and strategy use). Prospective memory (Memory of Intention Test) and HR-QoL (FAMS) were also improved. However, no significant differences were observed between groups for the CVLT-II, the MFQ, and Actual Reality, which is an ecological assessment of functional performances during an internet-based task. Therefore, the hypothesis that this rehabilitation technique could be transferred to other cognitive functions, notably the executive functions, could not be confirmed.

In a recent pilot study, Self-GEN strategy was associated with two other strategies of memory and learning rehabilitation, space learning (SL), and retrieval practice (RP), in the Strategy-based Training to Enhance Memory (STEM) program [38]. This pilot study, with limited power (20 patients), did not show a significant superiority of the STEM program to the control intervention (non-training program), on the primary endpoint (CVLT-II) and other objective tests of memory (BVMT-R), but it did show some encouraging trends and positive results on HR-QoL (FAMS and Short-Form 36 [SF-36]).

\subsubsection{Multiple Domains}

Three blinded RCTs were devoted to the individual rehabilitation of cognitive disorders in several domains in MS: two against no intervention and one against a non-specific training program $[16,20,34]$.

The first study compared a 13-week individual NP rehabilitation program in a large sample of patients (99) with no intervention [16,40]. The primary endpoint of this study was the subjective perception of deficits using the Perceived Deficits Questionnaire (PDQ). A greater improvement of this score was observed in the rehabilitated group compared to the control group. Regarding the NP tests, such as the Symbol Digit Modalities Test (SDMT), which was the second primary outcome, and the other tests of the BriefRepeatable Battery (BRB), no significant improvement was observed, except for the Trial Making Test-A (TMT-A). Note that the intervention's total duration in this partially negative study was much lower than in the other two individual multidomain studies (780 min versus 3600 and $2250 \mathrm{~min}$ ).

Although with smaller numbers (43 and 35), the other two studies had significantly better results on the NP tests [20,34]. The first study proposed an individual rehabilitation protocol called MS-Line!, combining written exercises and computer-assisted exercises over a period of 24 weeks compared to no intervention [20]. Although a primary analysis criterion was not specified in the report, the study showed significant results in favor of the treated group for improvement on several NP tests: TMT-A, Spatial Recall Test (SPART-10-36), Letter-Number Sequencing (LNS), Word List Generation (WLG), and the Boston Naming test (BNT). However, the results were negative for some other tests (TMT-B, SDMT, Selective Reminding Test (SRT), and digit span) and HR-QoL scales. Daily cognitive functioning was not studied. No long-term follow-up was done.

The REACTIV study was launched to demonstrate the superiority of a specific CR program (REACTIV) over nonspecific intervention (NSI) for NP assessment, virtual reality cognitive testing, and daily cognitive functioning in MS [34]. It was a single-blind RCT comparing these two interventions in patients with MS selected based on CI at specific tests of IPS, WM, and EF. Both programs included 50 individual sessions administered three times a week for 17 weeks. The specific intervention was tailored to patients' deficits. The primary endpoint was NP assessment of IPS, attention, EF, and WM. Secondary endpoints 
included ecological assessment by tasks in a virtual reality environment (Urban Daily $\operatorname{Cog}^{\circledR}$ ) and daily cognitive functioning assessment. More NP scores improved significantly in the active group and several NP scores, alertness and divided attention, and the ecological assessments improved significantly more after specific CR than after NSI. Lastly, SCR improved daily cognitive functioning. Most improvements were maintained 4 months after the end of the intervention. However, HR-Qol was not shown to be improved. The study showed the interest of an individualized and intense intervention including a metacognitive approach. It was also the first to show a transfer in ecological tasks. However, the study was underpowered for showing a larger effect on cognitive function. The study was performed in a real-world setting with rehabilitation by speech therapists in city practice.

\subsection{Group Interventions}

Five blinded RCTs have been reported about group intervention, but one of them mixed CT and group intervention [30]. The studies are difficult to compare because of the different rehabilitation programs and the different lengths of rehabilitation.

Among the four group intervention-only studies, the largest study was negative [35]. This study was a single blind study against no intervention and chose the psychological component of the Multiple Impact Scale (MSIS), a HR-QoL scale, as the primary endpoint at 12 months, which was no more improved in the treated group-although a significant small effect was seen at six months. There were no significant differences in NP tests between the two groups. The three other studies showed some positive results $[28,29,32]$. The first study showed improvements in EM and EF tests, but this study did not identify a primary endpoint [28]. The second study focused solely on the assessment of working memory and showed significant improvement after the group intervention compared to either a group that received relaxation or a group that received no intervention [29]. A double-blind multicenter study compared group rehabilitation to a sham intervention (non-training) [32]. In a fairly large sample of patients selected on the basis of the presence of a cognitive deficit, the main evaluation criterion was EM measured by the Selective Reminding Test (SRT), and a significant difference was observed in the learning curve between the treated group and the control group. WM also improved. However, there was no improvement in the other functions studied (EF, IPS). There was also no positive effect on HR-QoL.

The last study is difficult to interpret because of a mixed intervention combining group management and CT using Lumosity software [30]. Moreover, patients could be included regardless of the presence or absence of $\mathrm{CI}$, and the percentage of patients who were actually cognitively impaired at inclusion is not reported. Overall, the study was negative, the primary endpoint, the Everyday Problems Test-Revised, a self-administered questionnaire, was negative, and among the NP tests, only two were improved: the CVLT and the PASAT. This negative outcome could possibly be due to a significant improvement in the control group treated by a computer game.

\subsection{Computerized Multidomain Rehabilitation}

The largest group of studies devoted to CR in MS is devoted to CT (Tables 1 and 5).

The RCTs concerning multidomain CR, including attention, IPS, EF, and WM, have been developed in three categories of setting: in institutions, at home with telerehabilitation (online), and at home offline. The duration and number of CT rehabilitation sessions varied from one study to another with sessions lasting 30 to $60 \mathrm{~min}$ with a frequency of 2 to 3 per week for a duration of 4 to 12 weeks and a total duration of 300 to 2160 min.

Different software programs were used, the most frequent being RehaCom ${ }^{\circledR}$. Up to 2021, eight studies have been published with this software, and four blinded RCTs were selected for this review $[15,22,25,36]$. The first study used different modules of the RehaCom ${ }^{\circledR}$ software, delivered in the institution, in the control group than the module evaluated, and this study did not show a clear difference between these modalities [15]. However, the three most recent studies provide fairly consistent evidence for the effectiveness of rehabilitation using RehaCom $[22,25,36]$. The first study, although of limited 
power, showed an efficacy of RehaCom ${ }^{\circledR} \mathrm{CT}$ at home on the SDMT [22]. The patients were treated by the divided attention, WM, and visuo-spatial memory modules of RehaCom, and the control group received placebo intervention (projection of DVD). The second study compared rehabilitation using RehaCom's IPS/attention, memory, and EF modules with no treatment in the institution [25]. Although this endpoint was not clearly predefined in the paper, a Time $x$ Group interaction effect was shown by ANOVA on composite $z$ scores for different cognitive domains (verbal EM, attention, fluency, and IPS). The effects were maintained at six months. The same team performed a study in patients with secondary progressive MDS (SPMS) using RehaCom ${ }^{\circledR}$ CT at home compared to sham CT [36]. The $\mathrm{CT}$ was performed at home by the patient in the presence of a caregiver and with regular monitoring by a psychologist. This study had the longest total rehabilitation time with the RehaCom ${ }^{\circledR}$ software (1080 min). The primary endpoint (change in Brief International Cognitive Assessment for MS, BICAMS) was achieved with a greater significant improvement of the three BICAMS tests in the treated group as compared to the sham group. HRQoL was also more improved (EQ-5D).

Several studies have looked at other CT programs in MS and are summarized in Table 5.

Three studies used the Posit Science software programs Brain $\mathrm{HQ}^{\circledR}[16,30]$ and Insight ${ }^{\circledR}$ [12]. The study by Charvet et al. [16] was the first to show the feasibility of telerehabilitation using a research version of Brain $\mathrm{HQ}^{\circledR}$ compared to a computer game. This study showed the feasibility of such a telerehabilitation but with relatively modest results in terms of efficiency (improvement of the composite score without improvement of individual NP scores). A recent study also proposed TR using Brain $\mathrm{HQ}^{\circledR}$ but with a lower duration $(480 \mathrm{~min}$ ) [30]. No predefined primary endpoint was mentioned in the report, but several NP tests (EM and EF) were more improved in the treated group than in the control group without any intervention. In the last study, two software programs were used (Insigt from Posit Science and n-back from Brain Twister) [19]. Patients were not selected according to CI. The PASAT (one of the primary outcomes) was the only test more improved in the treated group than in the control group.

Two studies used the Attention processing Training Program (ATP) $[17,24]$. The study by Amato et al. was the first to show the feasibility of a home-based program [17]. Although this program was developed to improve the different components of attention, only the PASAT test has been improved, highlighting its relatively limited effectiveness. The second study that used APT, in a sample of patients not selected according to the presence of a CI, was negative [24].

The last four studies used different programs (Nintendo, Cogni-Track, Speed of Processing Training (SPT), and Cogmed) $[18,21,26,39]$. One study compared the telerehabilitation of WM using Cogmed to no intervention and did not show any difference between groups, but some tests improved only in the treated group [39]. A pilot study about SPT in 20 patients did not show any difference between groups on the primary outcomes (NP tests), but IPS improved only in the treated group, and a significant difference between groups was observed for a measure of daily cognitive functioning, the Timed Instrumental Activities of Daily Living Test [26]. The two other studies did not identify a predefined primary endpoint in their report but showed some effects of the Cogni-Track program on some NP tests of EM, WM, IPS, and EF [21] and of Dr. Kawashima's Brain Training ${ }^{\circledR}$ (Nintendo) on SMT and the Stroop test [18].

\section{Discussion and Conclusions}

The review performed in 2013 by Amato et al. [1] concluded that CR research has methodologic limitations in MS, and although targeted interventions to improve memory and learning show promise, they require further studies. The Cochrane Review published shortly after underlined the low-level evidence for positive effects of neuropsychological rehabilitation in MS [6]. The methodological quality of studies concerning CR in MS has improved significantly compared to the period before 2013. Most of the studies reviewed met most of the quality criteria: $\mathrm{RCT}$, blinded, control group with intervention, 
defined primary endpoint, assessment of efficacy on neuropsychological tests, but also daily cognitive functioning and HR-QoL.

Altogether, the studies about memory individual CR support the efficacy of m-SMT for improving learning in MS patients. This technique is able to improve verbal episodic memory and HR-QoL. There is some evidence of the efficiency of daily cognitive functioning, but there is no evidence of transfer in other domains. More data are necessary to support other methods of memory individual CR.

The studies about individual multidomain $\mathrm{CR}$ are difficult to compare, considering the differences between interventions. The first study used a short CR program (780 min) and did show an improvement in perceived deficits but not in NP scores $[16,40]$. The second study showed a significant effect on various NP measures but not on HR-QOL [20]. The third study was the only one in this category with an active control group receiving non-specific cognitive training, but was probably underpowered [34]. However, it showed the significant positive superiority of the specific training on several measures of attention but also evidence of improved cognitive functioning and improvement in an ecological task. This study also shows the feasibility of $\mathrm{CR}$ in a daily life setting. The results of these two studies support the efficacy of individual specific CR in MS, but they also emphasize the importance of the commitment required from patients who must devote many hours each week, which limits the feasibility of the program for some of them. Overall, most studies of specialized individual rehabilitation have given positive and encouraging results, but this type of rehabilitation requires significant human resources and a significant time investment by patients.

Although group rehabilitation requires fewer resources overall and is easier to implement, the number of studies that have been devoted to it is quite limited, and limited conclusions can be drawn so far. The largest study [35] was negative, one study was positive but without a specified primary endpoint [28], one study was focused only on WM [29], and one combined group CR and CT [30]. The more consistent results are provided by the study of Brissart et al. [32], showing a significant improvement in episodic and working memory. However, no effect on other cognitive domains, daily life cognitive functioning, or HR-QoL was demonstrated.

The large number of studies about CT is probably due to the ease of this technique. The possibility of implementing this $\mathrm{CT}$ at home and with telerehabilitation is also very promising. The variety of software used in these studies makes it difficult to compare them. RehaCom has been the most used. Four studies used this software in this review. Three of them showed very encouraging results $[22,25,36]$ on NP scores. Interestingly, the latter [36], performed in SPMS, showed a significant improvement in HR-Qol. All in all, these studies on RehaCom tend to show the effectiveness of this rehabilitation program in MS but leave several important questions unanswered: its use in telerehabilitation, its effectiveness on specific sub-domains according to the cognitive domain most affected, the interest of adding individual rehabilitation sessions including in particular a meta-cognitive approach, its effectiveness on daily cognitive functioning assessed by ecological tests, and its effect on quality of life in RRMS.

The other studies of CT with different programs (APT, PS, SPT) showed various results. As a result of the different software used, it is difficult to draw general conclusions, but some of them demonstrated the feasibility of home-based CR $[17,18,21]$ and of telerehabilitation $[23,37,39]$. The evidence of the efficacy of telerehabilitation is still limited, but the results encourage further studies in this field. The development of computerized assessment methods for cognitive disorders in MS, which have been the subject of a recent review [42], may help the deployment of telerehabilitation.

Although a demonstration of the effectiveness of multidomain CR, in particular on quality of life and transfer to cognitive domains different from those trained, is lacking, several studies show the feasibility of individual CR in daily life settings and home telerehabilitation using CT. A certain level of efficacy has been established regarding NP outcomes and in several studies on daily cognitive functioning. 
Funding: No funding for this work.

Institutional Review Board Statement: Not applicable.

Informed Consent Statement: Not applicable.

Conflicts of Interest: The author declares no conflict of interest in relation with this paper.

\section{References}

1. Amato, M.P.; Langdon, D.; Montalban, X.; Benedict, R.H.B.; DeLuca, J.; Krupp, L.; Thompson, A.; Comi, G. Treatment of cognitive impairment in multiple sclerosis: Position paper. J. Neurol. 2012, 260, 1452-1468. [CrossRef]

2. Ruet, A.; Deloire, M.; Hamel, D.; Ouallet, J.-C.; Petry, K.; Brochet, B. Cognitive impairment, health-related quality of life and vocational status at early stages of multiple sclerosis: A 7-year longitudinal study. J. Neurol. 2012, 260, 776-784. [CrossRef]

3. Kalb, R.; Beier, M.; Benedict, R.H.; Charvet, L.; Costello, K.; Feinstein, A.; Gingold, J.; Goverover, Y.; Halper, J.; Harris, C.; et al. Recommendations for cognitive screening and management in multiple sclerosis care. Mult. Scler. J. 2018, 24, 1665-1680. [CrossRef]

4. $\quad$ Landmeyer, N.C.; Bürkner, P.C.; Wiendl, H.; Ruck, T.; Hartung, H.P.; Holling, H.; Meuth, S.G.; Johnen, A. Disease-modifying treatments and cognition in relapsing-remitting multiple sclerosis: A meta-analysis. Neurology 2020, 94, e2373-e2383. [CrossRef]

5. Brochet, B.; Ruet, A. Cognitive Impairment in Multiple Sclerosis with Regards to Disease Duration and Clinical Phenotypes. Front. Neurol. 2019, 10, 261. [CrossRef] [PubMed]

6. $\quad$ Benedict, R.H.; DeLuca, J.; Phillips, G.; LaRocca, N.; Hudson, L.D.; Rudick, R.; Multiple Sclerosis Outcome Assessments Consortium. Validity of the Symbol Digit Modalities Test as a cognition performance outcome measure for multiple sclerosis. Mult. Scler. 2017, 23, 721-733. [CrossRef]

7. Rao, S.M.; Losinski, G.; Mourany, L.; Schindler, D.; Mamone, B.; Reece, C.; Kemeny, D.; Narayanan, S.; Miller, D.M.; Bethoux, F.; et al. Processing speed test: Validation of a self-administered, iPadR-based tool for screening cognitive dysfunction in a clinic setting. Mult. Scler. 2017, 23, 1929-1937. [CrossRef] [PubMed]

8. Ruet, A.; Deloire, M.S.A.; Charre-Morin, J.; Hamel, D.; Brochet, B. A new computerised cognitive test for the detection ofinformation processing speed impairment in multiple sclerosis. Mult. Scler. 2013, 19, 1665-1672. [CrossRef] [PubMed]

9. Benedict, R.H.B.; Cox, D.; Thompson, L.L.; Foley, F.; Weinstock-Guttman, B.; Munschauer, F. Reliable screening for neuropsychological impairment in multiple sclerosis. Mult. Scler. J. 2004, 10, 675-678. [CrossRef] [PubMed]

10. Langdon, D.W.; Amato, M.P.; Boringa, J.; Brochet, B.; Foley, F.; Fredrikson, S.; Hämäläinen, P.; Hartung, H.-P.; Krupp, L.; Penner, I.-K.; et al. Recommendations for a Brief International Cognitive Assessment for Multiple Sclerosis (BICAMS). Mult. Scler. J. 2011, 18, 891-898. [CrossRef] [PubMed]

11. Rao, S.M.; Leo, G.J.; Bernardin, L.; Unverzagt, F. Cognitive dysfunction in multiple sclerosis: I. Frequency, patterns, and prediction. Neurology 1991, 41, 685-691. [CrossRef]

12. Benedict, R.H.; Cookfair, D.; Gavett, R.; Gunther, M.; Munschauer, F.; Garg, N.; Weinstock-Guttman, B. Validity of the minimal assessment of cognitive function in multiple sclerosis (MACFIMS). J. Int. Neuropsychol. Soc. 2006, 12, 549-558. [CrossRef] [PubMed]

13. Rosti-Otajärvi, E.M.; Hämäläinen, P.I. Neuropsychological rehabilitation for multiple sclerosis. Cochrane Database Syst. Rev. 2014, 11, CD009131. [CrossRef]

14. Chiaravalloti, N.D.; Moore, N.B.; Nikelshpur, O.M.; DeLuca, J. An RCT to treat learning impairment in multiple sclerosis: The MEMREHAB trial. Neurology 2013, 81, 2066-2072. [CrossRef] [PubMed]

15. Cerasa, A.; Gioia, M.C.; Valentino, P.; Nisticò, R.; Chiriaco, C.; Pirritano, D.; Tomaiuolo, F.; Mangone, G.; Trotta, M.; Talarico, T.; et al. Computer-assisted cognitive rehabilitation of attention deficits for multiple sclerosis: A randomized trial with $\mathrm{fMRI}$ correlates. Neurorehabil. Neural Repair. 2013, 27, 284-295. [CrossRef] [PubMed]

16. Rosti-Otajärvi, E.; Mäntynen, A.; Koivisto, K.; Huhtala, H.; Hämäläinen, P. Neuropsychological rehabilitation has beneficial effects on perceived cognitive deficits in multiple sclerosis during nine-month follow-up. J. Neurol. Sci. 2013, 15, 154-160. [CrossRef] [PubMed]

17. Amato, M.P.; Goretti, B.; Viterbo, R.G.; Portaccio, E.; Niccolai, C.; Hakiki, B.; Iaffaldano, P.; Trojano, M. Computer-assisted rehabilitation of attention in patients with multiple sclerosis: Results of a randomized, double-blind trial. Mult. Scler. 2014, 20, 91-98. [CrossRef]

18. De Giglio, L.; De Luca, F.; Prosperini, L.; Borriello, G.; Bianchi, V.; Pantano, P.; Pozzilli, C. A low-cost cognitive rehabilitation with a commercial video game improves sustained attention and executive functions in multiple sclerosis: A pilot study. Neurorehabil. Neural Repair. 2015, 29, 453-461. [CrossRef]

19. Hancock, L.M.; Bruce, J.M.; Bruce, A.S.; Lynch, S.G. Processing speed and working memory training in multiple sclerosis: A double-blind randomized controlled pilot study. J. Clin. Exp. Neuropsychol. 2015, 37, 113-127. [CrossRef]

20. Gich, J.; Freixenet, J.; Garcia, R.; Vilanova, J.C.; Genís, D.; Silva, Y.; Montalban, X.; Ramió-Torrentà, L. A randomized, controlled, single-blind, 6-month pilot study to evaluate the efficacy of MS-Line! A cognitive rehabilitation programme for patients with multiple sclerosis. Mult. Scler. J. 2015, 21, 1332-1343. [CrossRef] 
21. Pedullà, L.; Brichetto, G.; Tacchino, A.; Vassallo, C.; Zaratin, P.; Battaglia, M.A.; Bonzano, L.; Bove, M. Adaptive vs. non-adaptive cognitive training by means of a personalized App: A randomized trial in people with multiple sclerosis. J. Neuroeng. Rehabil. 2016, 13, 88. [CrossRef]

22. Campbell, J.; Langdon, D.; Cercignani, M.; Rashid, W. A Randomised Controlled Trial of Efficacy of Cognitive Rehabilitation in Multiple Sclerosis: A Cognitive, Behavioural, and MRI Study. Neural Plast. 2016, 2016, 4292585. [CrossRef] [PubMed]

23. Charvet, L.E.; Yang, J.; Shaw, M.T.; Sherman, K.; Haider, L.; Xu, J.; Krupp, L.B. Cognitive function in multiple sclerosis improves with telerehabilitation: Results from a randomized controlled trial. PLoS ONE 2017, 12, e0177177. [CrossRef]

24. Grasso, M.G.; Broccoli, M.; Casillo, P.; Catani, S.; Pace, L.; Pompa, A.; Rizzi, F.; Troisi, E. Evaluation of the Impact of Cognitive Training on Quality of Life in Patients with Multiple Sclerosis. Eur. Neurol. 2017, 78, 111-117. [CrossRef] [PubMed]

25. Messinis, L.; Nasios, G.; Kosmidis, M.H.; Zampakis, P.; Malefaki, S.; Ntoskou, K.; Nousia, A.; Bakirtzis, C.; Grigoriadis, N.; Gourzis, P.; et al. Efficacy of a Computer-Assisted Cognitive Rehabilitation Intervention in Relapsing-Remitting Multiple Sclerosis Patients: A Multicenter Randomized Controlled Trial. Behav. Neurol. 2017, 2017, 5919841. [CrossRef] [PubMed]

26. Chiaravalloti, N.D.; Goverover, Y.; Costa, S.L.; DeLuca, J. A Pilot Study Examining Speed of Processing Training (SPT) to Improve Processing Speed in Persons with Multiple Sclerosis. Front. Neurol. 2018, 9, 685. [CrossRef] [PubMed]

27. Goverover, Y.; Chiaravalloti, N.; Genova, H.; DeLuca, J. A randomized controlled trial to treat impaired learning and memory in multiple sclerosis: The self-GEN trial. Mult. Scler. J. 2017, 24, 1096-1104. [CrossRef]

28. Mani, A.; Chohedri, E.; Ravanfar, P.; Mowla, A.; Nikseresht, A. Efficacy of group cognitive rehabilitation therapy in multiple sclerosis. Acta Neurol. Scand. 2018, 137, 589-597. [CrossRef] [PubMed]

29. Mousavi, S.; Zare, H.; Etemadifar, M.; Neshatdoost, H.T. Memory rehabilitation for the working memory of patients with multiple sclerosis (MS). J. Clin. Exp. Neuropsychol. 2017, 40, 405-410. [CrossRef] [PubMed]

30. Stuifbergen, A.K.; Becker, H.; Perez, F.; Morrison, J.; Brown, A.; Kullberg, V.; Zhang, W. Computer-assisted cognitive rehabilitation in persons with multiple sclerosis: Results of a multi-site randomized controlled trial with six month follow-up. Disabil. Health J. 2018, 11, 427-434. [CrossRef]

31. Krch, D.; Lequerica, A.; Arelis, A.A.; Barajas, B.V.R.; Arango-Lasprilla, J.C.; Chiaravalloti, N.D. Efficacy of the Spanish modified Story Memory Technique in Mexicans with multiple sclerosis: A pilot randomized controlled trial. NeuroRehabilitation 2019, 45, 349-358. [CrossRef]

32. Brissart, H.; Omorou, A.Y.; Forthoffer, N.; Berger, E.; Moreau, T.; De Seze, J.; Morele, E.; Debouverie, M. Memory improvement in multiple sclerosis after an extensive cognitive rehabilitation program in groups with a multicenter double-blind randomized trial. Clin. Rehabil. 2020, 34, 754-763. [CrossRef]

33. Chiaravalloti, N.D.; Moore, N.B.; DeLuca, J. The efficacy of the modified Story Memory Technique in progressive MS. Mult. Scler. J. 2019, 26, 354-362. [CrossRef]

34. Lamargue, D.; Koubiyr, I.; Deloire, M.; Saubusse, A.; Charre-Morin, J.; Moroso, A.; Coupé, P.; Brochet, B.; Ruet, A. Effect of cognitive rehabilitation on neuropsychological and semiecological testing and on daily cognitive functioning in multiple sclerosis: The REACTIV randomized controlled study. J. Neurol. Sci. 2020, 415, 116929. [CrossRef] [PubMed]

35. Lincoln, N.B.; Bradshaw, L.E.; Constantinescu, C.S.; Day, F.; Drummond, A.E.; Fitzsimmons, D.; Harris, S.; Montgomery, A.A.; das Nair, R.; CRAMMS Trial Collaborative Group. Cognitive rehabilitation for attention and memory in people with multiple sclerosis: A randomized controlled trial (CRAMMS). Clin. Rehabil. 2020, 34, 229-241. [CrossRef]

36. Messinis, L.; Kosmidis, M.H.; Nasios, G.; Konitsiotis, S.; Ntoskou, A.; Bakirtzis, C.; Grigoriadis, N.; Patrikelis, P.; Panagiotopoulos, E.; Gourzis, P.; et al. Do Secondary Progressive Multiple Sclerosis patients benefit from Computer- based cognitive neurorehabilitation? A randomized sham controlled trial. Mult. Scler. Relat. Disord. 2020, 39, 101932. [CrossRef]

37. Vilou, I.; Bakirtzis, C.; Artemiadis, A.; Ioannidis, P.; Papadimitriou, M.; Konstantinopoulou, E.; Aretouli, E.; Messinis, L.; Nasios, G.; Dardiotis, E.; et al. Computerized cognitive rehabilitation for treatment of cognitive impairment in multiple sclerosis: An explorative study. J. Integr. Neurosci. 2020, 19, 341-347. [CrossRef] [PubMed]

38. Chiaravalloti, N.D.; Moore, N.B.; Weber, E.; DeLuca, J. The application of Strategy-based Training to Enhance Memory (STEM) in multiple sclerosis: A pilot RCT. Neuropsychol. Rehabil. 2019, 31, 231-254. [CrossRef] [PubMed]

39. Blair, M.; Goveas, D.; Safi, A.; Marshall, C.; Rosehart, H.; Orenczuk, S.; Morrow, S.A. Does cognitive training improve attention/working memory in persons with MS? A pilot study using the Cogmed Working Memory Training program. Mult. Scler. Relat. Disord. 2021, 49, 102770. [CrossRef]

40. Mäntynen, A.; Rosti-Otajärvi, E.; Koivisto, K.; Lilja, A.; Huhtala, H.; Hämäläinen, P. Neuropsychological rehabilitation does not improve cognitive performance but reduces perceived cognitive deficits in patients with multiple sclerosis: A randomised, controlled, multi-centre trial. Mult. Scler. 2014, 20, 99-107. [CrossRef]

41. Chiaravalloti, N.D.; DeLuca, J.; Moore, N.B.; Ricker, J.H. Treating learning impairments improves memory performance in multiple sclerosis: A randomized clinical trial. Mult. Scler. 2005, 11, 58-68. [CrossRef] [PubMed]

42. Wojcik, C.M.; Beier, M.; Costello, K.; DeLuca, J.; Feinstein, A.; Goverover, Y.; Gudesblatt, M.; Jaworski, M.; Kalb, R.; Kostich, L.; et al. Computerized neuropsychological assessment devices in multiple sclerosis: A systematic review. Mult. Scler. J. 2019, 25, 1848-1869. [CrossRef] [PubMed] 